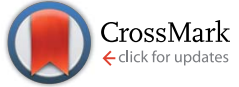

Cite this: RSC Adv., 2017, 7, 9465

Received 7th November 2016

Accepted 22nd January 2017

DOI: 10.1039/c6ra26434d

rsc.li/rsc-advances

\title{
Further exploring the feasibility of dendritic cells- targeted biomimetic Texosomes as a therapeutic and preventive tumor-vaccine
}

\begin{abstract}
Kexin Li, ${ }^{*}$ Shasha Chang, Xiuli Zhao and Dawei Chen
Targeting tumor cells with immunotherapy by a dendritic cells-targeted vaccination is a potential treatment option. In previous research, we preliminarily proved the immune activity of hTERT-HSP70 loaded DEC205McAb Texosomes on H22 hepato-carcinoma bearing mice. In this article, in order to further verify the feasibility of DEC205McAb Texosomes as a new type of anti-tumor vaccine in clinical treatment, we also inspected the vaccine's broad spectrum, the best immune period, the vaccine's preventability, and moreover the influence of immune inhibitors on this vaccine. Anti-tumor immune responses were evaluated by determining the tumor volume, mice survival rate, and the IL-12 and IFN $\gamma$ antibody levels. Our results suggest that DEC205McAb Texosomes could also efficiently inhibit the tumor growth of S180 ascites sarcoma bearing mice with a 7 day immune period, and we also found that DEC205McAb Texosomes as an anti-tumor vaccine could achieve not only good therapeutic treatment but also effective preventive performance. In conclusion, we hope that dendritic cellstargeted anti-tumor vaccines could become a new approach in the field of cancer treatment and prevention.
\end{abstract}

\section{Introduction}

Perhaps you have thought about the question: why can the immune system prevent a lot of diseases, but can't inhibit tumor growth in vivo? The answer is very simple, and is that the lack of an effective anti-tumor response is one of the main reasons for cancer progression..$^{1-3}$ After all, most tumor cells derive from the human body's self-proteins; the immune system will not easily see them as foreign objects, and therefore will not take the initiative to attack them.

The ideal tumor therapy would enhance the body's natural defense against tumor cells, and at the same time destroy the actual tumor. In past years, a number of strategies have been used to enhance the immune responses of tumor vaccines, including dendritic cells-based vaccines, which are firstly separated from peripheral blood of tumor patients and pulsed with cytokine genes or tumor associated antigen peptide in vitro, and then retransfused into the body through veins..$^{4-6}$ But once dendritic cells are not mature enough, the various types of cytokines will be weaker expression or no expression, as a result $\mathrm{T}$ cells can't effectively be stimulated and activated, and more seriously induce anergy or tolerance of $\mathrm{T}$ cells directly.

Chimeric Antigen Receptor T-cell technology (CART) is much more potent than any other immune-based treatments being studied. ${ }^{7-9}$ These engineered CART cells are then grown in the

School of Pharmacy, Shenyang Pharmaceutical University, Liaoning Province, China. E-mail: kexinbest1128@163.com; Tel: +862423986293 laboratory until they number in the billions. The proliferating CART cells are then infused into the patient. After the infusion, if all goes as planned, the $\mathrm{T}$ cells multiply in the patient's body and, with guidance from their engineered receptor, recognize and kill cancer cells that harbor the antigen on their surfaces. ${ }^{10}$ But the infused $\mathrm{T}$ cells could release cytokines, and if there is a rapid and massive release of cytokines into the bloodstream, which can lead to dangerously high fevers and precipitous drops in blood pressure. Moreover, clinical trials demonstrate that CART therapy is only more effective for leukemia.

At present, these two kinds of effective immune treatment methods both need to extract the patient's immune cells "dendritic cells or $\mathrm{T}$ cells" from peripheral blood and further process in vitro, which means very high cost and poor patient tolerance. ${ }^{11,12}$ So how to effectively immunize cancer patients against specific tumor antigen by generating anti-tumor $\mathrm{T}$ cells and inducing T-cell mediated cell immunity is an urgent problem to be solved. ${ }^{13}$ What we should do first is how to "tell" the characteristics of the tumor antigen to immune system in vivo, in addition allow body's immunocytes to actively search, locate, attack and kill tumor cells. ${ }^{14}$

In the article we have published before, we indicate that hTERT-HSP70 loaded DEC205McAb Texosomes could enhance tumor specific immune responses on $\mathrm{H} 22$ tumor bearing mice, which could trigger strong cytotoxic $\mathrm{T}$ lymphocyte reaction and consequently inhibit the tumor growth. ${ }^{15}$ However, whether finally DC-targeted biomimetic Texosomes could be used in clinical as vaccination purposes in vivo, it is necessary to carry 
out a lot of further research and some critical variables are still to be optimized, just as verifying the vaccine's broad spectrum, investigating the best immune period of vaccine administration, exploring the vaccine's preventability, in addition discussing the influence of immune inhibitors on this vaccine.

\section{Experimental}

\section{Materials}

Phosphatidylcholine (PC) was purchased from Avanti Polar Lipids (Shanghai, China), cholesterol $(\mathrm{CH})$ was purchased from Tianjin Bodi Chemical (China), dioleoyl phosphoethanolamine (DOPE), 3-( $N$-( $N, N$-dimethylaminoethane)carbamoyl)cholesterol (DC Chol), DEC205 monoclonal antibody (DEC205McAb) were obtained from Sigma (UK), antigen peptide complex hTERT-HSP70 was self-prepared in our lab.

\section{Administration preparation}

Dendritic cells targeted biomimetic Texosomes loading hTERTHSP70 as universal tumor antigen were prepared by microemulsion and micelle assembling method just as we described in previously published article. ${ }^{16}$ DEC205 monoclonal antibodies were then covalently conjugated to the surface of Texosomes to obtain DEC205 monoclonal antibodies coupled biomimetic Texosomes (DEC205McAb Texosomes). ${ }^{17}$ The mean diameter of DEC205McAb Texosomes was $92.73 \pm 7.69 \mathrm{~nm}$ and the zeta potential was $+21.0 \pm 4.48 \mathrm{mV}$. The encapsulation efficiency was $87.27 \pm 5.55 \%$ and the conjugation efficiency of DEC205McAb Texosomes was $59.77 \pm 6.72 \%$.

\section{In vivo immunization studies}

Establishment of S180 tumor-bearing mice models. Healthy female KM mice (weight, 18-22 g) were purchased from animal center of Shenyang Pharmaceutical University in Liaoning province, China. Animals were housed separately with free access to food and water according to SPF standards. Animals were maintained in accordance with the guidelines of Regulations for the Administration of Affairs Concerning Experimental animals, China, and approved by the Institutional Animal Care and Use Committee (IACUC) of Shenyang Pharmaceutical University. The tumor-bearing mice were produced by inoculating a suspension of $\mathrm{S} 180$ cells $\left(3 \times 10^{7}\right.$ cells per $\left.\mathrm{ml}\right)$ subcutaneously into the right axillary fossa. When the tumor reached a volume of $50 \mathrm{~mm}^{3}$, the mice were respectively divided into different groups with six mice in each group. Tumor length $(a)$ and width $(b)$ were measured with a caliper every 3 days, and tumor volume $\left(V, \mathrm{~mm}^{3}\right)$ was calculated by: $V=a b^{2} / 2$. Determination of the antibody levels of IL-12 and IFN $\gamma$.

Mice blood samples were collected by retro-orbital puncture after different days of primary immunization and sera was separated and stored at $-70{ }^{\circ} \mathrm{C}$ for later analysis. The antibody levels of IL-12 and IFN $\gamma$ were detected by ELISA kit in triplicate. IL-12 and IFN $\gamma$ concentrations were determined from a standard curve plotted using known concentrations of standard IL12 and IFN $\gamma$.

\section{Study of immune spectrum}

Six groups of mice were immunized 3 times at 0 th, 7 th and 14 th day by abdominal subcutaneous with saline, HSP70 solution, hTERT solution, hTERT-HSP70 solution, Texosomes and DEC205McAb Texosomes containing $100 \mu \mathrm{g} \mathrm{kg}^{-1}$ of antigen. Mice blood samples were collected at day 7, 14 and 21 by retroorbital puncture and sera was separated and stored at $-70{ }^{\circ} \mathrm{C}$ for later analysis to quantitatively detect the antibody levels of IL-12 and IFN $\gamma$ by ELISA.

\section{Study of immune period}

The S180 tumor bearing mice were randomly divided into four groups with six mice in each group. Three groups of mice were immunized three times respectively with 2, 4 and 7 days intervals by abdominal subcutaneous with DEC205McAb Texosomes containing $100 \mu \mathrm{g} \mathrm{kg}^{-1}$ of antigen, and the mice in control group was also administrated three times by saline in according with 7 days cycle. Mice blood samples were then collected at 7th, 14th and 21st day.

\section{Study of immune inhibitor}

The S180 tumor bearing mice were randomly divided into tacrolimus group, normal preparation group and saline group with six mice in each group. Mice in tacrolimus group were given $0.2 \mathrm{mg} \mathrm{kg}^{-1}$ tacrolimus suspension by oral administration from 0 th day to 20th day once every two days. Then tacrolimus group and normal preparation group were immunized three times respectively at 0 th, 7 th and 14 th day by abdominal subcutaneous with DEC205McAb Texosomes containing $100 \mu \mathrm{g}$ $\mathrm{kg}^{-1}$ of antigen, and saline group was immunized with saline according to the same immune period. Mice blood samples were also collected at 7 th, 14 th and 21 st day.

\section{Study of immune preventability}

In order to investigate whether DEC205McAb Texosomes has the function of preventive immunity, we respectively set up advanced immune group, normal immune group and saline group with six mice in each group. In advanced immune group, healthy mice were immunized three times with DEC205McAb Texosomes according to 4 days interval in advance, and then on the second day after the last immunization, mice were inoculated tumor by a suspension of $\mathrm{S} 180$ cells $\left(3 \times 10^{7}\right.$ cells per $\left.\mathrm{ml}\right)$ subcutaneously into the right axillary fossa. We chose this day as 0th day, then measured tumor volume every 3 days and furthermore recorded survival rate. In normal immune group and saline group, we directly selected tumor-bearing mice with the tumor volume above $50 \mathrm{~mm}^{3}$. And the mice in normal immune group and saline group were immunized respectively with DEC205McAb Texosomes and saline at 0th, 7th and 14th day, then we also measured tumor size every 3 days and recorded survival rate.

\section{Statistical analysis}

All values in the present study were expressed as the mean \pm standard error of the mean. Statistical analysis was performed 
using OriginPro 8.5. Differences between two groups were tested using a Student's $t$-test. Significant differences between the groups were expressed as follows: ${ }^{*} p<0.05,{ }^{* *} p<0.01$.

\section{Results and discussion}

\section{Verifying of broad spectrum}

We select two representative models of tumor bearing mice, respectively $\mathrm{S} 180$ ascites sarcoma and $\mathrm{H} 22$ hepato-carcinoma to discuss whether the new designed tumor vaccine loading universal tumor antigen peptide have broad therapeutic effect. Although sarcomas and carcinomas both belong to malignant tumors, their origins are different. The tumor originating from epithelial tissue is carcinoma and the tumor originating from mesenchymal tissue is sarcoma. There are many differences in the origin, incidence, age of onset, predilection site, metastasis pathway and prognosis effect. In previous article, we proved DEC205McAb Texosomes could inhibit tumor growth and prolong the survival of $\mathrm{H} 22$ tumor bearing mice. ${ }^{15}$ So in this study, we further carried out the immune experiments on S180 tumor bearing mice. Fig. 1 showed that DEC205McAb Texosomes could obviously inhibit the tumor growth and prolonged the survival period of tumor-bearing mice to 44 days compared with the other five groups, which indicates its excellent anti-tumor effect. IL-12 as a mature DCs' signature cytokine and IFN $\gamma$ as a CTL cytokine produced obviously higher levels, which showed strong tumor-specific cytotoxic $\mathrm{T}$ lymphocytes have been triggered and they would recognize the tumor cells and generate effective anti-tumor immune response. Just as professor Gillian Griffiths said, cytotoxic $\mathrm{T}$ cells have a pretty important role to play in keeping your body healthy. They move rapidly around their environment looking for cancerous cells. ${ }^{18}$ Once cytotoxic $\mathrm{T}$ cells recognize tumor antigen, they launch an attack by binding to the cell and injecting it with poisonous molecules called cytotoxins. After the cytotoxins are injected into the cancer cells, its fate is sealed and it withers and dies. Then $\mathrm{T}$ cells move on, hungry to find another tumor cells. So we conclude that biomimetic DEC205McAb Texosomes loaded hTERT-HSP70 have therapeutic effects on many kinds of tumors and the new carriers have the potential to become a universal vaccine against tumor with the same tumor antigen.

\section{Investigating of immune period}

The production and strength of immune response are closely related to the immune period. Against different antigens, the best time for producing antibodies is also different. In this article, we investigated the effects of different immune cycles on the immune response in mice. Fig. 2(A) showed that within 15 days, obvious tumor growth inhibition effect could be obtained by three groups of different immune periods as compared with saline group. But to 2 day group, which initially could limit the tumor growth, but after 15 days, the inhibition effect gradually decreased and the tumor volume increased significantly. At the same time, the tumor-bearing mice in 2 day group showed severe immunological response beyond the tolerance range of mice, and some symptoms of drowsiness, weight loss and

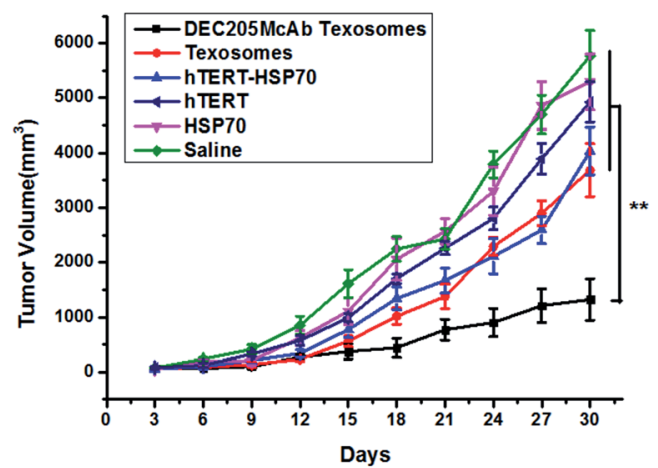

(A)

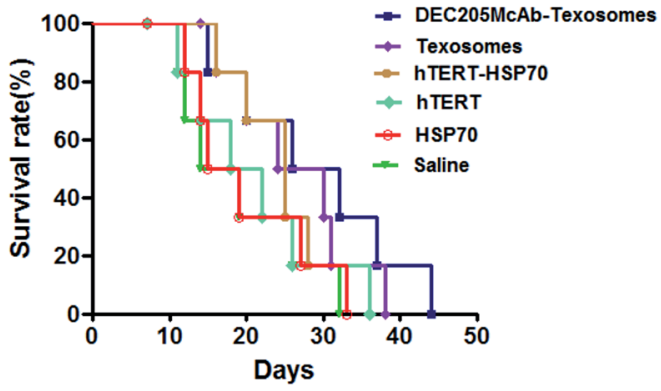

(B)

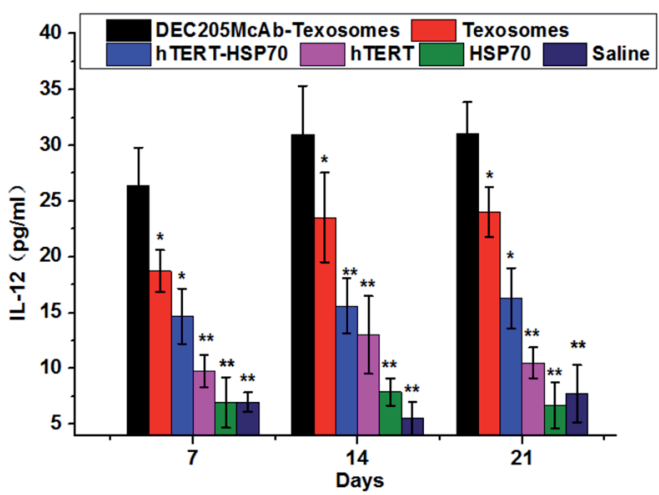

(C)

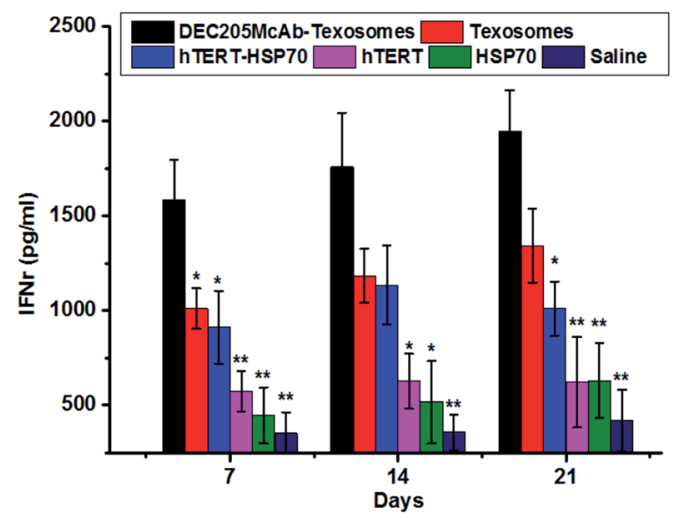

(D)

Fig. 1 The tumor volume (A), survival curves (B), the IL-12 levels (C) and IFN $\gamma$ levels (D) of S180 tumor bearing mice treated with saline, HSP70 solution, hTERT solution, hTERT-HSP70 solution, Texosomes and DEC205McAb Texosomes. Statistical difference between two groups was reported as: $* p<0.05, * * p<0.01$ vs. DEC205McAb Texosomes group. 


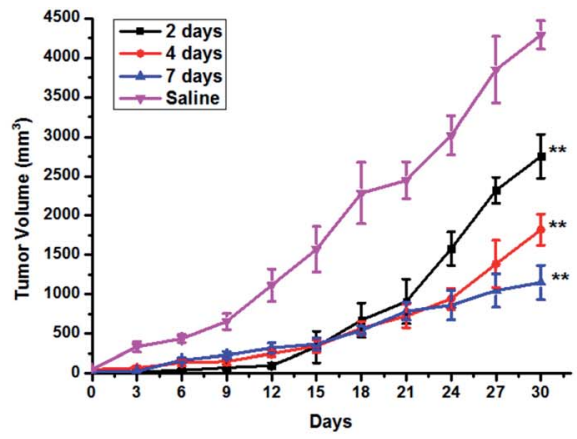

(A)

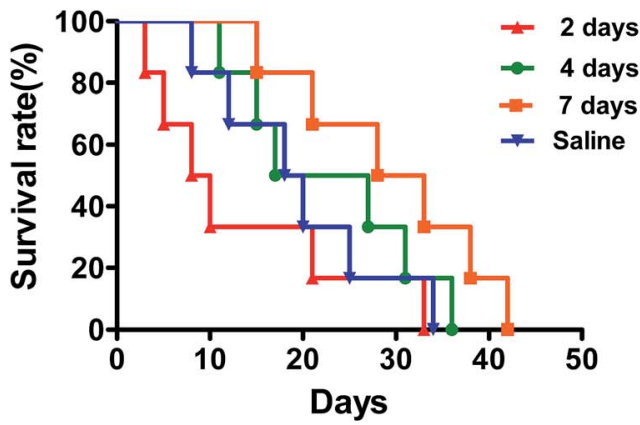

(B)

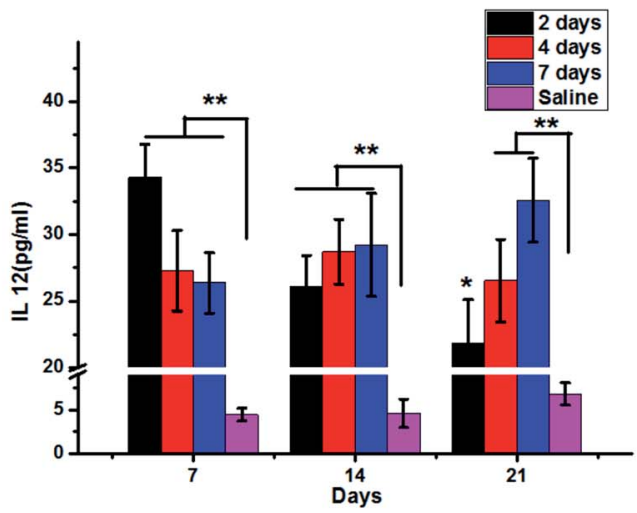

(C)

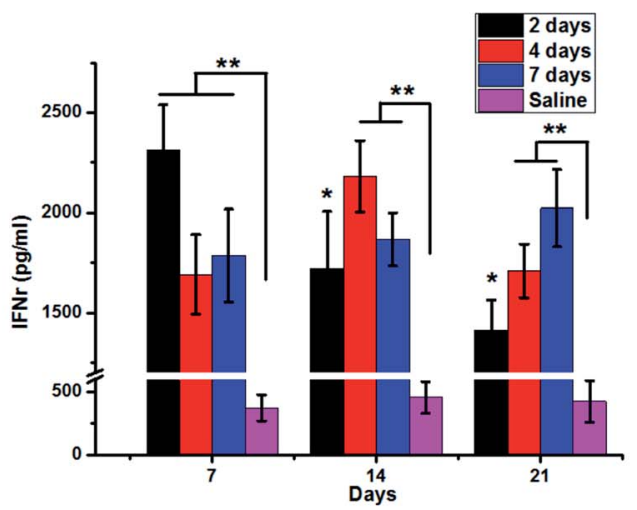

(D)

Fig. 2 The tumor volume (A), survival curves (B), the IL-12 levels (C) and IFN $\gamma$ levels (D) of S180 tumor-bearing mice immunized according to different immune periods of 2 days, 4 days and 7 days, meantime saline group was chose as negative control by 7 days interval. Statistical difference was reported as: ${ }^{*} p<0.05, * * p<0.01$ vs. saline group. apathetic state occurred from the second immunization, meanwhile mice died at 3rd, 5th, 8th and 10th day one by one. The status of mice for 4 day group was also not good, just as slowed to weakness, reduced feeding and fur was not glory. Compared with the above two groups, the tumor bearing mice in 7 day group were in better condition, the survival time was longer, and the maximum length could be extended to 42nd day.

We simultaneously found that whether it was for IL-12 or IFN $\gamma$ immune levels, there was a significant difference between any test group and the control group. The IL-12 and IFN $\gamma$ levels of 2 day group were $34.30 \pm 3.88 \mathrm{pg} \mathrm{ml}^{-1}$ and $2314.68 \pm 224.1$ $\mathrm{pg} \mathrm{ml}^{-1}$ at 7 th day, $26.07 \pm 2.34 \mathrm{pg} \mathrm{ml}^{-1}$ and $1722.36 \pm 285.3 \mathrm{pg}$ $\mathrm{ml}^{-1}$ at 14 th day, $21.85 \pm 3.21 \mathrm{pg} \mathrm{ml}^{-1}$ and $1411.76 \pm 151.6 \mathrm{pg}$ $\mathrm{ml}^{-1}$ at 21 st day, which told us that when the immunization period was 2 days, the tumor-bearing mice produced a very high level of immunity just at 7th day, moreover the rapid growth of immune factors was beyond the scope of the immune tolerance of mice, therefore, a serious immune response occurred. But after 7 days, the immune levels reduce rapidly, and the lower immune factors could not inhibit tumor growth. With the extension of the immune interval period, at 7 th day we could see that the IL-12 and IFN $\gamma$ levels of the 4 day and 7 day groups were lower compared with those of the 2 day group, and the highest immune levels occurred at 14 th day to the 4 day group, then decreased gradually, which is consistent with the result of the tumor volume. But to 7 day group, the immune factor levels in the serum of immuned mice gradually increased or maintained stable accompany with immunization times, so the tumorbearing mice were well tolerated and the survival period was longer.

Just as you known, the generation of an immune response needed time. After 1-2 weeks of the antigen getting into the body, the proliferation of $\mathrm{T}$ cells reached the peak, then $\mathrm{T}$ cells came into the systole period and a few of them turned into memory T cells. ${ }^{19}$

To 2 day and 4 day groups, the higher immune levels could get from the beginning, but with the effector $\mathrm{T}$ cell apoptosis, the antibody levels significantly drop afterwards. But to mice of 7 day group, the body's immune system had enough time to produce a moderate immune response and also maintain a high level of immunity for a long time, so we chose the immune period as 7 days. At the same time, we also think that the best immune period is not only for S180 tumor cells, but also appropriate for other tumor cells as long as they express the same antigen peptide-Telomerase reverse transcriptase (hTERT), because the body's immune reaction against the same antigen should be similar, and the only difference is just the different targeting tumor cells to kill.

\section{Influence of immune inhibitor}

Tacrolimus is one kind of widely used immunosuppressive agents, and it could inhibit the maturation of $\mathrm{T}$ lymphocytes mainly through the inhibition of interleukin-2 (IL-2) release. ${ }^{\mathbf{2 0 - 2 2}}$ In this research, tacrolimus is given to these mice as an immunosuppressant, and we would like to investigate whether 
the ability of DEC205McAb Texosomes to reduce the tumor volume was affected. The results of Fig. 3(A) and (B) told us that once the immune inhibitor was used, DEC205McAb Texosomes would lost its anti-tumor immune function, and there was no difference in tumor size and survival rate between the tacrolimus group and saline group. The output can clearly suggest that the role of immune activation for DEC205McAb Texosomes is achieved by activation of $\mathrm{T}$ lymphocytes.

There is a significant difference between the normal preparation group and saline group on the levels of IL-12 and IFN $\gamma$, but the tacrolimus group was consistent with the saline group at 7 th, 14th and 21st day, they both kept the IL-12 and IFN $\gamma$ at very low levels all the time, that is to say the immunosuppressive agent could interfere with the immune function of the immune system and prevent the acquired immune response.

As was known to all, the production of IL-12 is a mature symbol of dendritic cells. ${ }^{23}$ The low immune levels of mice in tacrolimus group proved that dendritic cells remain blocked at an immature stage of development, that is, they only have strong phagocytic ability but not the antigen presenting ability to T cells. While IFN $\gamma$ as a Th1 signature cytokine could activate cytotoxic $\mathrm{T}$ lymphocyte to recognize the tumor cells and generate effective anti-tumor immune response. ${ }^{24}$ And the low IFN $\gamma$ level of tacrolimus group told us that tumor-specific cytotoxic $\mathrm{T}$ lymphocytes could not be activated and so failed to specific kill the tumor cells by releasing perforin. In conclusion, the above results proved that DEC205McAb Texosomes inhibit the cancer growth by the method of triggering strong tumor-specific cytotoxic $\mathrm{T}$ lymphocyte reaction.

\section{Exploring of preventive immunization}

From the above experiments, we have demonstrated that DEC205McAb Texosomes could effectively inhibit tumor growth and prolong the survival period, so it could be used as therapeutic anti-tumor vaccine. At the same time, we are much more interested in whether DEC205McAb Texosomes have the preventive effect on tumor. After inoculating with S180 tumor cells, 2 mice in advanced immune group had no growth tumors, and the other 4 mice still remained small tumor volume, basically no growth. And the tumor volume of mice in normal immune group immunized by DEC205McAb Texosomes showed an increasing trend in general, but compared with the saline group, the tumor size could be effectively controlled, just as Fig. 4(A) showed. Survival curve told us that in normal immune group, the survival period was prolonged to 42 days, while the advanced immune group could be increased to 53 days. Meanwhile, the mice in these two groups were both in good condition and did not appear excessive immune symptoms.

The above results indicated that DEC205McAb Texosomes have the effect of preventive immunization, and the production or growth of tumor can be effectively suppressed after immunization in advance. We deduced that the reason was that once DEC205McAb Texosomes was injected, it would stimulate the immune system to produce tumor antigen-specific cytotoxic $\mathrm{T}$ lymphocytes, so later even if tumor antigen was inoculated into

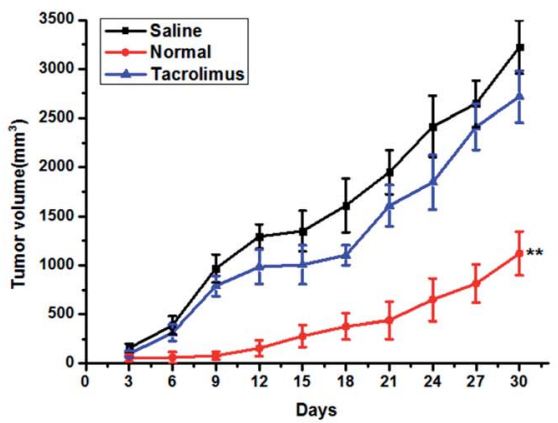

(A)

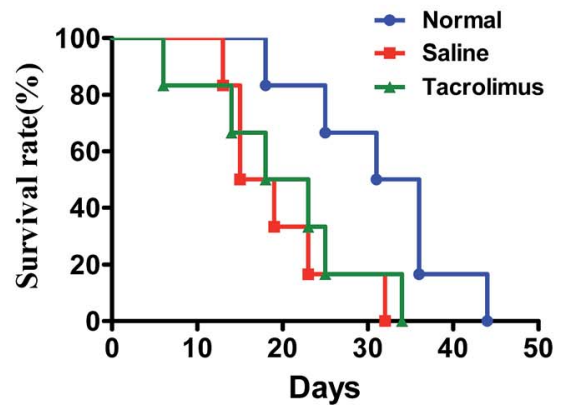

(B)

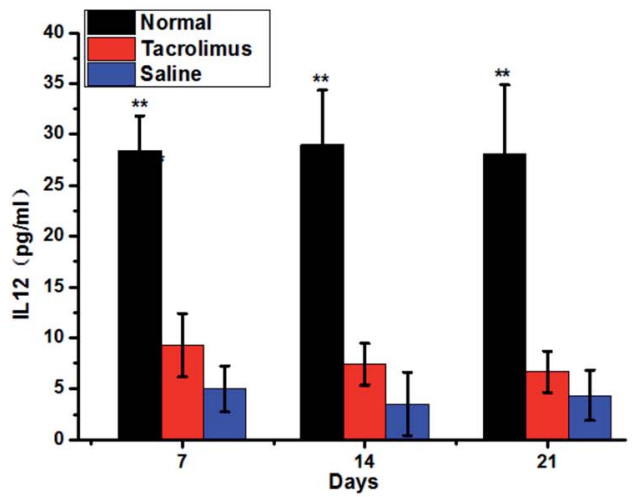

(C)

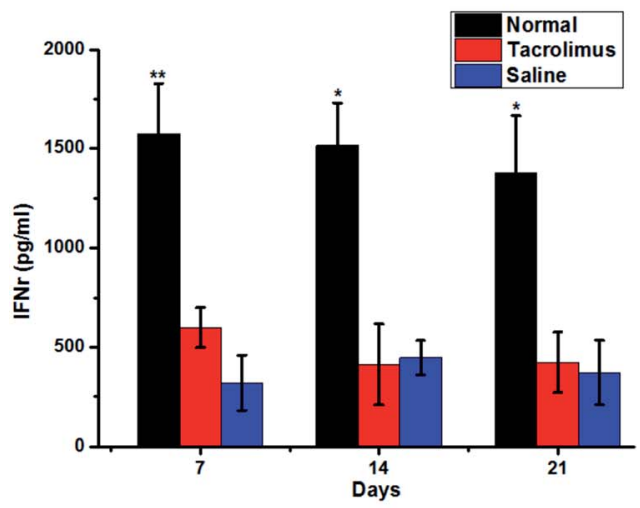

(D)

Fig. 3 The tumor volume (A), survival curves (B), the IL-12 levels (C) and IFN $\gamma$ levels (D) of S180 tumor bearing mice immunized by normal preparation group (normal), tacrolimus group (tacrolimus) and saline group (saline). Statistical difference between two groups was reported as: $* p<0.05, * * p<0.01$ vs. saline group. 


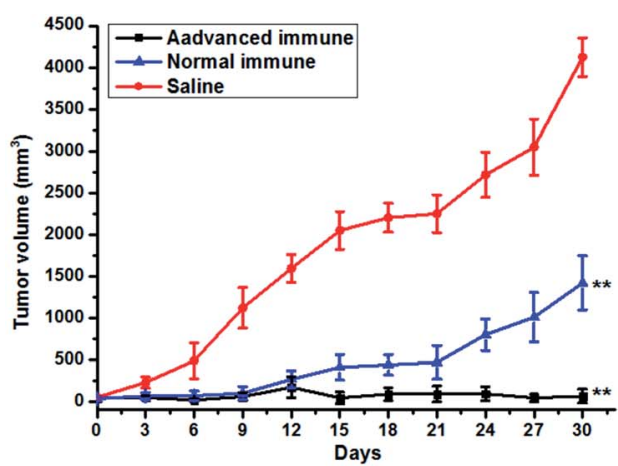

(A)

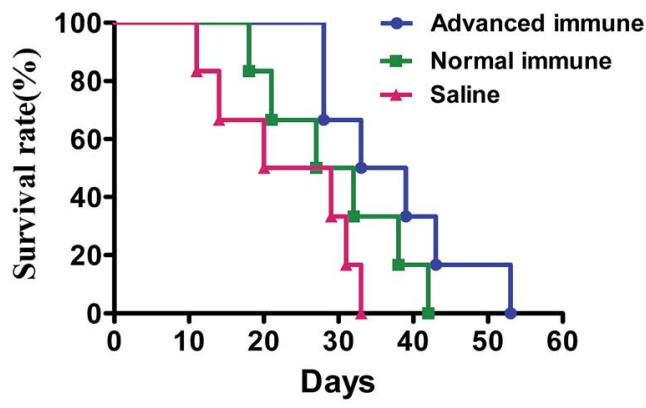

(B)

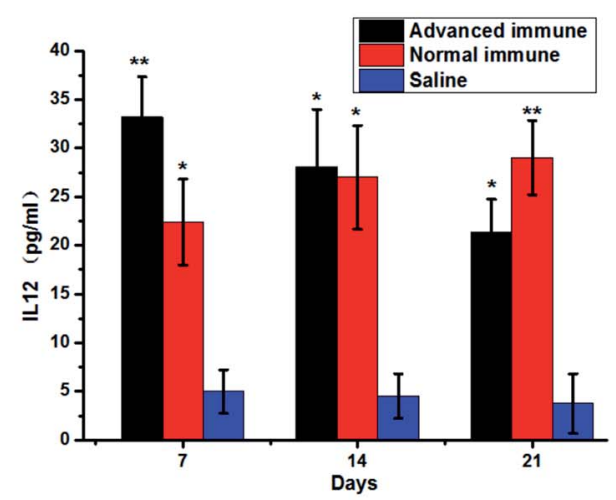

(C)

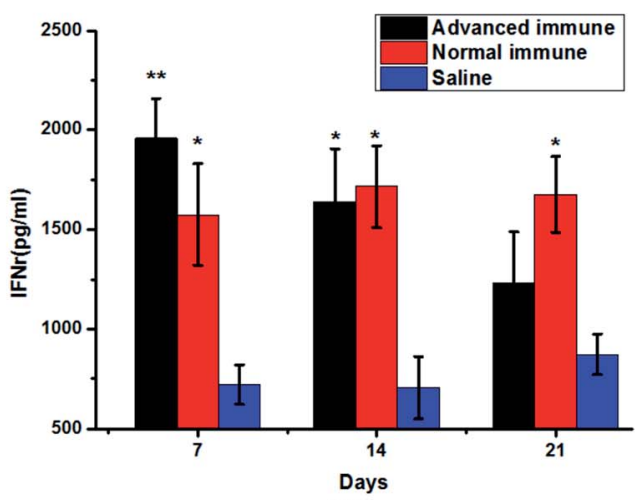

(D)

Fig. 4 The tumor volume (A), survival curves (B), the IL-12 levels (C) and IFN $\gamma$ levels (D) of S180 tumor bearing mice immunized by the way of advanced immune with DEC205McAb Texosomes (advanced immune), normal immune respectively with DEC205McAb Texosomes (normal immune) and saline (saline). Values were considered significant when $* p<0.05$, and ${ }^{* *} p<0.01$ vs. saline group. mice, cytotoxic $\mathrm{T}$ lymphocytes could actively search for tumor cells and accurately kill them by releasing the perforin, therefore, prevented tumor growth and decreased the growth rate. But the mice in normal immune group were immunized after the tumor formation, and solid tumors have already existed and formed during the course of the immune system producing immune response, so we could see that even if the DEC205M$\mathrm{cAb}$ Texosomes could control the speed of tumor growth to a great extent and effectively inhibit the development of tumor, but it still could not completely wipe out the solid tumors. Therefore, DEC205McAb Texosomes has a certain role in the prevention and treatment of tumors, especially it can be used as a prophylactic vaccine against cancer.

Compared with the normal immune group, at 7th day, mice in advanced immune group have showed very high levels of IL12 and IFN $\gamma$. The reasons perhaps were that if DEC205McAb Texosomes was immunized three times in advance, mice would produce adaptive immune response. At the recognition stage, $\mathrm{T}$ cells recognized the tumor antigen presented by dendritic cells, and then in the activation and proliferation stage, $\mathrm{T}$ cells gradually induced into tumor antigen-specific cytotoxic $\mathrm{T}$ lymphocytes, meantime released the immune factors. ${ }^{25,26}$ That was to say, before S180 tumor cells were inoculated, the in vivo immune response was already formed, mature dendritic cells and cytotoxic T lymphocytes had already existed. So, when the mice were injected with $\mathrm{S} 180$ suspension, the body immediately entered the effective stage, a part of memory $\mathrm{T}$ cells was awakened and generated protective immune responses, meantime cytotoxic $\mathrm{T}$ lymphocytes already presented in vivo would accurately track and ultimately destroy tumor cells through specific recognition of tumor antigens. So our results showed that, as compared with solid tumors, cytotoxic $\mathrm{T}$ lymphocytes have stronger lethality to free tumor cells, that is to say, once new tumor cells appear in vivo, the activated CTL will immediately look for and eventually kill them, which means that the preparation has great potential as a preventive vaccine. More significant, as we known, while nowadays surgical treatment of a localized tumor is often successful, the treatment of metastatic tumors remains a severe challenge, DEC205McAb Texosomes we prepared here has the great advantage of finding free tumor cells, so they could be used as a strong auxiliary method of surgical treatment of tumor, effective prevent postoperative metastasis of tumor.

\section{Conclusion}

The ability to immunize cancer patients against specific tumor antigens by generating anti-tumor $\mathrm{T}$ cells and inducing $\mathrm{T}$-cell mediated cell immunity marked the beginning of immunotherapy in cancer treatment. A number of strategies have been used to enhance the immune responses of tumor vaccine, in this article we further tracked the feasibility of dendritic cellstargeted biomimetic Texosomes as therapeutic and preventive tumor-vaccine. The results told us that (1) DEC205McAb Texosomes could inhibit tumor growth of mice with S180 ascites sarcoma and $\mathrm{H} 22$ hepato-carcinoma, meantime prolong the survival of tumor bearing mice which proved the vaccine have 
broad spectrum. (2) For the universal tumor antigen-telomerase reverse transcriptase, the optimal immune period of DEC205McAb Texosomes for KM mice was 7 days, which not only could ensure a better living condition, but also high levels of immune factors. (3) The immune-stimulating effect of DEC205McAb Texosomes was achieved by activation of $\mathrm{T}$ lymphocytes, because immune-suppressant tacrolimus could make the body appear immune tolerance state. (4) DEC205M$\mathrm{cAb}$ Texosomes also had the effect of preventive immunity, which could effectively suppress tumor formation or inhibit tumor growth to a great extent. At present, surgical resection operation is the best treatment method to cure tumor, but it still has a high recurrence rate, even to some early found tumors or the radical resection tumors, the recurrence rate is still high, seriously affecting the effects of short-term and long-term operation. This research proved that DEC205McAb Texosomes can not only achieve the effective prevention or early treatment to a variety of tumors, but also can be used as auxiliary means of surgical treatment, eliminate metastases and some free tumor cells, which has prominent effect on preventing recurrence after operation and tumor metastasis. In a word, DEC205McAb Texosomes would open a new chapter in the prevention and treatment of cancer.

\section{Acknowledgements}

We are grateful to financial support by the National Natural Science Foundation of China (Grant No. 81302721), Natural Science Foundation of Liaoning Province (No. 201602696) and general project of Education Department of Liaoning Province (No. L2014383).

\section{References}

1 N. K. Mehta, K. D. Moynihan and D. J. Irvine, Cancer Immunol. Res., 2015, 3, 836-843.

2 J. M. Vyas, A. G. Vander Veen and H. L. Ploegh, Nat. Rev. Immunol., 2008, 8, 607-618.

3 P. A. Roche and K. Furuta, Nat. Rev. Immunol., 2015, 15, $203-$ 216.

4 K. Palucka and J. Banchereau, Immunity, 2013, 39, 38-48.

5 J. J. Glass, D. Yuen, J. Rae, et al., Nanoscale, 2016, 8, 82558265.
6 K. K. Phua, S. K. Nair, K. W. Leong, et al., Nanoscale, 2014, 6, 7715-7729.

7 M. B. Geyer and R. J. Brentjens, Cytotherapy, 2016, 18(11), 1393-1409.

8 Z. G. Ahmed, S. E. Naggar and N. Ahmed, Cytotherapy, 2016, 18(11), 1382-1392.

9 S. Gill, M. V. Maus and D. L. Porter, Blood Rev., 2016, 30(3), 157-167.

10 M. B. Geyer and R. J. Brentjens, Cytotherapy, 2016, 18, 13931409.

11 M. Isabelle, D. Alterr and M. Eyrich, Cytotherapy, 2016, 18, 1146-1161.

12 S. Mocellin, S. Mandruzzato, V. Bronte, et al., Lancet Oncol., 2004, 5(11), 681-689.

13 R. M. Prins, H. Soto, V. Konkankit, et al., Clin. Cancer Res., 2011, 17(6), 1603-1615.

14 M. G. Kim, J. Y. Park and Y. Shon, Asian J. Pharm. Sci., 2014, 9, 227-235.

15 Z. Y. Wang, S. S. Chang and K. X. Li, $R S C A d v ., 2016,6$, 55819-55824.

16 K. X. Li, S. S. Chang and Z. Y. Wang, Int. J. Pharm., 2015, 491, 105-112.

17 G. Bendas, A. Krause, U. Bakowsky, et al., Int. J. Pharm., 1999, 181, 79-93.

18 G. M. Griffiths and J. C. Stinchcombe, J. Cell Biol., 2010, 189(3), 399-406.

19 D. T. Utzschneider, M. Charmoy, V. Chennupati, et al., Immunity, 2016, 45(2), 415-427.

20 J. J. Z. Leon, I. H. Fisac, S. Guerrero, et al., Transplant Immunol., 2016, 36, 9-13.

21 W. Lei, C. Q. Yu, H. Q. Lin, et al., Asian J. Pharm. Sci., 2013, 8(6), 336-345.

22 Y. Ren, Y. M. Yang, J. Yang, et al., Int. Immunopharmacol., 2014, 21, 247-254.

23 S. W. Lee, H. J. Park and K. S. Lee, Biochem. Biophys. Res. Commun., 2015, 461, 86-94.

24 T. D. Fernandez, J. R. Pearson and M. P. Leal, Biomaterials, 2015, 43, 1-12.

25 N. Kibbi, O. Sobolev, M. Girardi, et al., Transfus. Apher. Sci., 2016, 55(1), 146-152.

26 M. D. Charette, A. Marabelle and R. Houot, Eur. J. Cancer, 2016, 68, 134-147. 